\title{
Syntheses and Physical-Chemical Properties \\ of Phenylvinylidene Complexes with MnPt Core, \\ Containing Diphenylphosphine Ligand \\ at the Platinum Atom
}

\author{
Oleg S. Chudin*a, \\ Victor V. Verpekin ${ }^{\mathrm{a}}$, Alexander A. Kondrasenko ${ }^{\mathrm{a}}$, \\ Nina I. Pavlenko and Anatoly I. Rubaylo ${ }^{a, b}$ \\ IInstitute of Chemistry and Chemical Technology SB RAS \\ FRC "Krasnoyarsk Science Center SB RAS" \\ 50/24 Akademgorodok, Krasnoyarsk, 660036, Russia \\ ${ }^{b}$ Siberian Federal University \\ 79 Svobodny, Krasnoyarsk, 660041, Russia
}

Received 12.02.2018, received in revised form 23.07.2018, accepted 06.09.2018

A synthetic approach to the new heterobinuclear $\mu$-phenylvinylidene complexes $\mathrm{Cp}(\mathrm{CO})_{2} \mathrm{MnPt}(\mu$ $\mathrm{C}=\mathrm{CHPh})\left(\mathrm{PPh}_{2} \mathrm{H}\right)(\mathrm{L})\left[\mathrm{L}=\mathrm{PPh}_{3}(1), \mathrm{P}\left(\mathrm{OPr}^{\mathrm{i}}\right)_{3}(2)\right]$, containing diphenylphosphine ligand at the platinum atom, was developed. The compounds were studied by IR- and NMR-spectroscopy. Their molecular structures were proposed based on IR, NMR spectroscopic data.

Keywords: manganese, platinum, vivylidene, heterometallic complexes, vinylidene complexes, carbonyl ligand, diphenylphosphine.

Citation: Chudin O.S., Verpekin V.V., Kondrasenko A.A., Pavlenko N.I., Rubaylo A.I. Syntheses and physical-chemical properties of phenylvinylidene complexes with $\mathrm{MnPt}$ core, containing diphenylphosphine ligand at the platinum atom, J. Sib. Fed. Univ. Chem., 2018, 11(3), 390-400. DOI: 10.17516/1998-2836-0085.

(C) Siberian Federal University. All rights reserved

* Corresponding author E-mail address: oschudin@mail.ru 


\section{Синтез и физико-химические свойства}

\section{фенилвинилиденовых комплексов с остовом MnPt, содержащих дифенилфосфиновый лиганд \\ при атоме платины}

О.С. Чудин ${ }^{\mathrm{a}}$, В.В. Верпекин А.А. Кондрасенко ${ }^{\mathrm{a}}$, Н.И. Павленко ${ }^{\mathrm{a}}$, А.И. Рубайло ${ }^{\mathrm{a},}$ ${ }^{a}$ Институт химии и химической технологии СО РАН ФИЦ «Красноярский научный иентр СО РАН» Россия, 660036, Красноярск, Академгородок, 50/24

${ }^{6}$ Сибирский федеральныий университет Россия, 660041, Красноярск, пр. Свободный, 79

Разработан метод синтеза новых биядерных $\mu$-винилиденовых комплексов типа $\mathrm{Cp}(\mathrm{CO})_{2} \operatorname{MnPt}(\mu-\mathrm{C}=\mathrm{CHPh})\left(\mathrm{PPh}_{2} \mathrm{H}\right)(\mathrm{L}) \quad\left[L=\mathrm{PPh}_{3} \quad(1), \mathrm{P}_{(\mathrm{OPr}}\right)_{3} \quad$ (2)], содержаших дифенилфосфиновый лиганд $P$ P ${ }_{2} H$ при атоме платины. Полученные соединения изучены методами ИК- и ЯМР-спектроскопии. На основе спектроскопических данных предложено строение комплексов.

Ключевые слова: марганеи, платина, винилиден, гетерометаллические комплексы, винилиденовые комплексы, карбонил, дифенилфосфин.

\section{Введение}

Координационные и металлоорганические соединения, содержащие в своих молекулах два и более разных атомов металлов, представляют как научный, так и практический интерес. Исследования структуры физико-химических свойств и закономерностей образования таких соединений вносят вклад в фундаментальные разделы современной химии, такие как теория строения и реакционной способности, теория процессов каталитического синтеза [1-6], а благодаря наличию в своем составе различных атомов металлов и функциональных групп такие полиметаллические комплексы могут обеспечивать возможность протекания двух и более стадий каталитических процессов в пределах одной молекулы и поэтому являются перспективными катализаторами в органической химии [7-12].

Один из методов синтеза подобных гетерометаллических комплексов и кластеров основан на способности моноядерных комплексов, содержащих непредельные углеводородные лиганды, селективно присоединять координационно- и электронно-ненасыщенные металлосодержащие фрагменты [13-18]. Данный подход успешно реализован нами для синтеза би- и трехъядерных гетерометаллических винилиденовых комплексов и кластеров [19-24]. Однако координационные свойства винилиденов существенно ограничены, так как по связи $\mathrm{C}=\mathrm{C}$ этого лиганда могут присоединяться только до трех координационно- и электронно- 
ненасыщенных металлосодержащих фрагментов [20, 24]. Это ограничение можно снять путем введения в лигандное окружение металлов молекул, способных дополнительно координировать атомы других металлов, например дифенилфосфинов [25-27]. В связи с этим нами изучена возможность внедрения в биядерные $\mathrm{MnPt} \mu$-винилиденовые комплексы дифенилфосфиновых лигандов.

\section{Экспериментальная часть}

Все операции по синтезу и выделению комплексов проводили в атмосфере аргона. Растворители (бензол, диэтиловый эфир, петролейный эфир, хлористый метилен) предварительно очищали от примесей, следов воды и кислорода путем перегонки в инертной атмосфере над соответствующими осушителями. Комплексы $\mathrm{Cp}(\mathrm{CO})_{2} \mathrm{MnPt}(\mu-\mathrm{C}=\mathrm{CHPh})(\mathrm{L})_{2}$ и $\mathrm{Cp}(\mathrm{CO})_{2} \operatorname{MnPt}(\mu-\mathrm{C}=\mathrm{CHPh})(\mathrm{CO})(\mathrm{L})\left(\mathrm{L}=\mathrm{PPh}_{3}, \mathrm{P}\left(\mathrm{OPr}^{\mathrm{i}}\right)_{3}\right)$ синтезированы по методикам [28, 29]. $\mathrm{B}$ экспериментах использован дифенилфосфин $\mathrm{PPh}_{2} \mathrm{H}$ (Acros) и октакарбонилдикобальт $\mathrm{Co}_{2}(\mathrm{CO})_{10}$ (Aldrich).

Спектры ИК измеряли на ИК Фурье-спектрометре Tensor 27 (Bruker, Германия). Спектры ЯMP ${ }^{1} \mathrm{H},{ }^{13} \mathrm{C}$ и ${ }^{31} \mathrm{P}$ регистрировали на Фурье-спектрометре ЯMP AVANCE III 600 (Bruker, Германия). Химические сдвиги в спектрах были определены относительно остаточных протонов дейтерированных растворителей и приведены в миллионных долях (м.д.). Константы спинспиновых взаимодействий указаны в герцах (Гц).

Комплексы $\mathrm{Cp}(\mathrm{CO})_{2} \mathrm{Mn}(\mu-\mathrm{C}=\mathrm{CHPh}) \mathrm{Pt}\left(\mathrm{PPh}_{2} \mathrm{H}\right)(\mathrm{L})\left(\mathrm{L}=\mathrm{PPh}_{3}, \mathrm{P}\left(\mathrm{OPr}^{\mathrm{i}}\right)_{3}\right)$ синтезированы по следующим методикам:

Реакияия комплекса $\mathrm{Cp}(\mathrm{CO})_{2} \mathrm{MnPt}(\mu-\mathrm{C}=\mathrm{CHPh})\left(\mathrm{PPh}_{3}\right)(\mathrm{CO})$ c $\mathrm{PPh}_{2} \mathrm{H}$

К раствору 0.02 г (0.026 ммоль) $\mathrm{Cp}(\mathrm{CO}){ }_{2} \mathrm{MnPt}(\mu-\mathrm{C}=\mathrm{CHPh})\left(\mathrm{PPh}_{3}\right)(\mathrm{CO})$ в 0.6 мл $\mathrm{CD}_{2} \mathrm{Cl}_{2}$ в ампуле ЯМР прибавили 4.6 мкл (0.027 ммоль) $\mathrm{PPh}_{2} \mathrm{H}$. Согласно спектрам ${ }^{1} \mathrm{H}$ ЯМР ${ }^{31} \mathrm{P}\left\{{ }^{1} \mathrm{H}\right\}$ реакционная смесь содержит два новых соединения: комплекс $\mathrm{Cp}(\mathrm{CO})_{2} \mathrm{MnPt}(\mu-\mathrm{C}=\mathrm{CHPh})\left(\mathrm{PPh}_{2} \mathrm{H}\right)\left(\mathrm{PPh}_{3}\right)(\mathbf{1})$ и комплекс $\mathrm{Cp}(\mathrm{CO}){ }_{2} \mathrm{MnPt}(\mu-\mathrm{C}=\mathrm{CHPh})(\mathrm{CO})\left(\mathrm{PPh}_{2} \mathrm{H}\right)(\mathbf{8})$ в соотношении $1: 0.8$, соответственно.

Комплекс $\mathrm{Cp}(\mathrm{CO})_{2} \mathrm{MnPt}(\mu-\mathrm{C}=\mathrm{CHPh})\left(\mathrm{PPh}_{2} \mathrm{H}\right)\left(\mathrm{PPh}_{3}\right)(\mathbf{1})$

ИК спектр $\left(\mathrm{CH}_{2} \mathrm{Cl}_{2}, \mathrm{~cm}^{-1}\right): 1923,1828$ (vCO);

${ }^{1} \mathrm{H}$ ЯMP $\left(\mathrm{CD}_{2} \mathrm{Cl}_{2}, 25^{\circ} \mathrm{C}\right) \delta: 4.31\left(\mathrm{c}, \mathrm{C}_{5} \mathrm{H}_{5}\right) ; 5.58$ (д, $\left.-\mathrm{P} \underline{\mathrm{H}}, J_{\mathrm{HP}}=350\right) ; 6.99-7.72(\mathrm{Ph}) ; 8.08\left(\right.$ д, $=\mathrm{C}^{2} \underline{\mathrm{HPh}_{2}}$ $\left.J_{\mathrm{PH}}=11.0\right)$;

${ }^{31} \mathrm{P}\left\{{ }^{1} \mathrm{H}\right\}$ ЯMР $\left(\mathrm{CD}_{2} \mathrm{Cl}_{2}, 25^{\circ} \mathrm{C}\right) \delta: 5.74$ (дд, $\left.\mathrm{Ph}_{2} \underline{\mathrm{P}}, J_{\mathrm{PP}}=17.1, J_{\mathrm{PtP}}=2470\right) ; 41.04$ (дд, $\mathrm{Ph}_{3} \underline{\mathrm{P}}, J_{\mathrm{PP}}=17.1$, $\left.J_{\mathrm{PtP}}=4243\right)$.

Комплекс $\mathrm{Cp}(\mathrm{CO})_{2} \mathrm{MnPt}(\mu-\mathrm{C}=\mathrm{CHPh})(\mathrm{CO})\left(\mathrm{PPh}_{2} \mathrm{H}\right)(\boldsymbol{8})$

${ }^{1} \mathrm{H}$ ЯМР $\left(\mathrm{CD}_{2} \mathrm{Cl}_{2}\right) \delta: 4.50\left(\mathrm{c}, \mathrm{C}_{5} \mathrm{H}_{5}\right) ; 5.77$ (д, -P$\left.\underline{\mathrm{H}}, J_{\mathrm{HP}}=293\right) ; 7.07-7.74(\mathrm{Ph}) ; 8.20\left(\mathrm{c},=\mathrm{C}^{2} \underline{\mathrm{HPh}_{2}}\right)$;

${ }^{31} \mathrm{P}\left\{{ }^{1} \mathrm{H}\right\}$ ЯMP $\left(\mathrm{CD}_{2} \mathrm{Cl}_{2}\right) \delta: 6.83$ (д, $\left.\mathrm{Ph}_{2} \mathrm{P}, J_{\mathrm{PtP}}=2540\right)$.

Синтез $\mathrm{Cp}(\mathrm{CO})_{2} \mathrm{MnPt}(\mu-\mathrm{C}=\mathrm{CHPh})\left(\mathrm{PPh}_{2} \mathrm{H}\right)\left[\mathrm{P}\left(\mathrm{OPr}_{3}\right)_{3}\right]$ (2)

К раствору 0.100 г (0.141 ммоль) $\mathrm{Cp}(\mathrm{CO})_{2} \operatorname{MnPt}(\mu-\mathrm{C}=\mathrm{CHPh})(\mathrm{CO})\left[\mathrm{P}\left(\mathrm{OPr}^{i}\right)_{3}\right](7)$ в 8 мл бензола при интенсивном перемешивании в атмосфере аргона добавляли 27 мкл (0.155 ммоль) $\mathrm{PPh}_{2} \mathrm{H}$. Реакционную смесь перемешивали в течение 5 мин, растворитель удаляли при пониженном давлении. К полученному остатку добавили 5 мл петролейного эфира и наносили на колонку $\mathrm{Al}_{2} \mathrm{O}_{3}(8 \times 2 \mathrm{~cm})$. Колонку элюировали петролейным эфиром и смесью гексан-бензол (2:1). Первая бесцветная зона содержала небольшое количество избытка $\mathrm{PPh}_{2} \mathrm{H}$. Из второй желтой зоны по- 
сле удаления растворителя и последующей кристаллизации полученного остатка из диэтилового эфира получено 0.079 г (65\%) $\mathrm{Cp}(\mathrm{CO})_{2} \mathrm{MnPt}(\mu-\mathrm{C}=\mathrm{CHPh})\left(\mathrm{PPh}_{2} \mathrm{H}\right)\left[\mathrm{P}\left(\mathrm{OPr}^{\mathrm{i}}\right)_{3}\right](\mathbf{1})$ в виде темнооранжевых кристаллов.

ИК спектр $\left(\mathrm{CH}_{2} \mathrm{Cl}_{2}, \mathrm{~cm}^{-1}\right): 1923,1828$ (v(CO)); $\left(\mathrm{KBr}, \mathrm{cm}^{-1}\right):$ 1926, 1829 (v(CO)).

${ }^{13} \mathrm{C}\left\{{ }^{1} \mathrm{H}\right\}$ ЯMP $\left(\mathrm{CD}_{2} \mathrm{Cl}_{2}, \mathrm{~T}=-15{ }^{\circ} \mathrm{C}\right) \delta: 23.7\left(\mathrm{c},-\mathrm{CH}_{3}\right) ; 23.9\left(\mathrm{c},-\mathrm{CH}_{3}\right) ; 70.8$ (д, - $\left.\mathrm{CH}, \mathrm{J}_{\mathrm{CPt}}=23.8\right)$; $84.6\left(\mathrm{c}, \underline{\mathrm{C}}_{5} \mathrm{H}_{5}\right), 123.8\left(\mathrm{c}, \mathrm{C}_{\mathrm{p}},=\mathrm{C}^{\mathrm{l}}=\mathrm{C}^{2} \mathrm{HPh}\right) ; 125.7\left(\mathrm{c}, \mathrm{C}_{\mathrm{o}},=\mathrm{C}=\mathrm{CHPh}\right) ; 128.3\left(\mathrm{c}, \mathrm{C}_{\mathrm{m}},=\mathrm{C}=\mathrm{CHPh}\right) ; 128.5$ (д, $\left.\underline{\mathrm{Ph}}_{2} \mathrm{P}, \mathrm{J}_{\mathrm{CP}}=8\right) ; 129.2$ (д, $\left.\mathrm{C}_{\text {кл }}, \underline{\mathrm{Ph}}_{2} \mathrm{P}, \mathrm{J}_{\mathrm{CP}}=42.9\right) ; 129.8\left(\mathrm{c}, \mathrm{C}_{\mathrm{p}}, \underline{\mathrm{Ph}}_{2} \mathrm{P}\right) ; 130.6$ (с, $\left.\mathrm{C}_{\mathrm{p}}, \underline{\mathrm{Ph}}_{2} \mathrm{P}\right) ; 132.4$ (д, $\mathrm{C}_{\mathrm{m}}$, $\left.\underline{\mathrm{Ph}_{2}}{ }_{2}, \mathrm{~J}_{\mathrm{CP}}=10.3\right) ; 132.5$ (д, $\left.\mathrm{C}_{\text {кл }}, \underline{\mathrm{Ph}_{2}} \mathrm{P}, \mathrm{J}_{\mathrm{CP}}=47.7\right) ; 135.5$ (д, $\left.\mathrm{C}_{\mathrm{m}}, \underline{\mathrm{Ph}_{2}} \mathrm{P}, J_{\mathrm{CP}}=11.1\right) ; 140.2$ (д, $=\mathrm{C}^{\mathrm{l}}=\underline{\mathrm{C}} \underline{2} \mathrm{HPh}$, $\left.J_{\mathrm{CPt}}=129.1\right) ; 143.6$ (д, $\left.\mathrm{C}_{\text {кл }}=\mathrm{C}^{\mathrm{l}}=\mathrm{C}^{2} \mathrm{HPh}, J_{\mathrm{CP}}=11.1\right) ; 229.7\left(\mathrm{c},-\mathrm{CO}_{\mathrm{t}}\right) ; 236.8$ (дд, $-\mathrm{CO}_{\mathrm{sb}}, J_{\mathrm{CP}}=12.7$, $\left.\mathrm{J}_{\mathrm{CPt}}=103.7\right) ; 261.2$ (дд, $=\underline{\mathrm{C}}=\mathrm{C}^{2} \mathrm{HPh}, J_{\mathrm{CP}}=62.0, J_{\mathrm{CPt}}=823.3$ ).

${ }^{1} \mathrm{H}$ ЯМР $\left(\mathrm{CD}_{2} \mathrm{Cl}_{2}, 25^{\circ} \mathrm{C}\right) \delta: 1.15$ (д, $\left.-\mathrm{CH}_{3}, J_{\mathrm{HP}}=5.5\right) ; 1.28$ (д, $\left.-\mathrm{CH}_{3}, J_{\mathrm{HP}}=5.7\right) ; 4.43\left(\mathrm{c}, \mathrm{C}_{5} \mathrm{H}_{5}\right) ; 4.79$

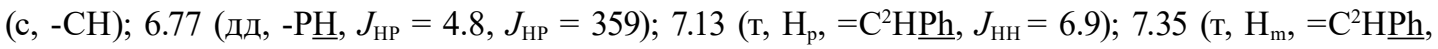
$\left.J_{\mathrm{HH}}=7.2\right) ; 7.48-7.55\left(\mathrm{~m}, \underline{\left.\mathrm{Ph}_{2} \mathrm{P}-\right) ; 7.79(д, ~} \mathrm{H}_{\mathrm{o}}=\mathrm{C}^{2} \mathrm{HPh}, J_{\mathrm{HH}}=7.32 \mathrm{~Hz}\right) ; 7.83\left(\mathrm{~T}, \mathrm{H}_{\mathrm{p}}, \underline{\mathrm{Ph}_{2}} \mathrm{P}-, J_{\mathrm{HH}}=8.8\right) ; 8.75$ (ддд, $\left.=\mathrm{C}^{2} \underline{\mathrm{HPh}}{ }_{2} J_{\mathrm{PH}}=14.9, J_{\mathrm{PtH}}=30.1\right)$.

${ }^{31} \mathrm{P}\left\{{ }^{1} \mathrm{H}\right\}$ ЯМР $\left(\mathrm{CD}_{2} \mathrm{Cl}_{2}, 25^{\circ} \mathrm{C}\right) \delta: 4.25$ (дд, $\left.\mathrm{Ph}_{2} \underline{\mathrm{P}}, J_{\mathrm{PP}}=11.4, J_{\mathrm{PtP}}=2252\right) ; 132.4$ (дд, $\underline{\mathrm{P}}\left(\mathrm{OPr}{ }^{\mathrm{i}}\right)_{3}, J_{\mathrm{PP}}=11.4$, $\left.J_{\mathrm{PtP}}=6714\right)$.

\section{Результаты и обсуждение}

С целью разработки методик синтеза биядерных $\mu$-винилиденовых комплексов типа $\mathrm{Cp}(\mathrm{CO})_{2} \mathrm{MnPt}(\mu-\mathrm{C}=\mathrm{CHPh})\left(\mathrm{PPh}_{2} \mathrm{H}\right)(\mathrm{L})\left(\mathrm{L}=\mathrm{PPh}_{3}(\mathbf{1}), \mathrm{P}\left(\mathrm{OPr}_{3}^{\mathrm{i}}\right)_{3}\right.$ (2)), содержащих дифенилфосфиновые лиганды при атоме платины, были изучены реакции комплексов $\mathrm{Cp}(\mathrm{CO})_{2} \operatorname{MnPt}(\mu-\mathrm{C}=\mathrm{CHPh})$ $\left.(\mathrm{L})_{2}\left(\mathrm{~L}=\mathrm{PPh}_{3}(3), \mathrm{P}(\mathrm{OPri})_{3}\right)(4)\right)$ с дифенилфосфином. Ранее было установлено [29], что трифенилфосфиновые и триизопропилфосфитные лиганды при атоме платины в этих комплексах могут последовательно замещаться на $\mathrm{P}\left(\mathrm{OPr}^{\mathrm{i}}\right)_{3}$ или $\mathrm{PPh}_{3}$ с первоначальным образованием смешанолигандного комплекса $\mathrm{Cp}(\mathrm{CO})_{2} \mathrm{Mn}(\mu-\mathrm{C}=\mathrm{CHPh}) \mathrm{Pt}\left[\mathrm{P}\left(\mathrm{OPr}_{3}\right)_{3}\right]\left(\mathrm{PPh}_{3}\right)(\mathbf{5})$ и дальнейшим образованием 3 и 4 (рис. 1).

Однако реакции комплексов 3 и $\mathbf{4}$ с дифенилфосфином не приводят к замещению фосфорсодержащих лигандов при атоме платины. Нами был предложен другой синтетический подход, предполагающий предварительную обработку 3 и 4 октакарбониломдикобальта с образованием комплексов $\mathrm{Cp}(\mathrm{CO})_{2} \mathrm{Mn}(\mu-\mathrm{C}=\mathrm{CHPh}) \mathrm{Pt}[\mathrm{L}](\mathrm{CO})\left(\mathrm{L}=\mathrm{PPh}_{3}(\mathbf{6}),\left[\mathrm{P}\left(\mathrm{OPr}^{\mathrm{i}}\right)_{3}\right](7)\right)$. Введенная карбонильная группа при атоме платины является более лабильной и может с легкостью замещаться на другие лиганды $[29,30]$. Действительно, реакция 7 с $\mathrm{PPh}_{2} \mathrm{H}$ приводит к селективному замещению группы СО и образованию 2 (рис. 2).
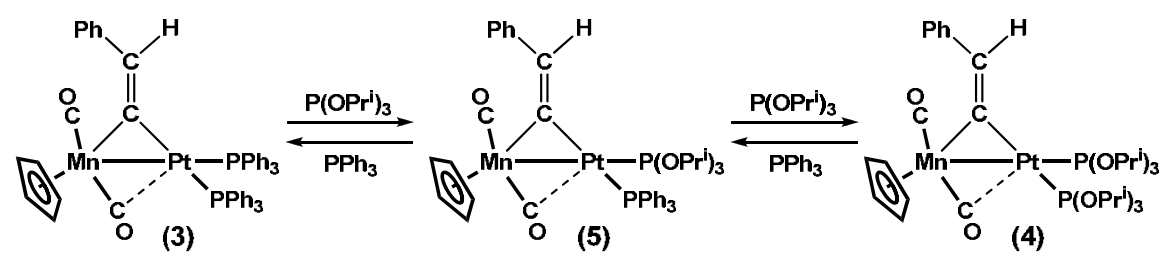

Рис. 1

Fig. 1

$$
-393-
$$




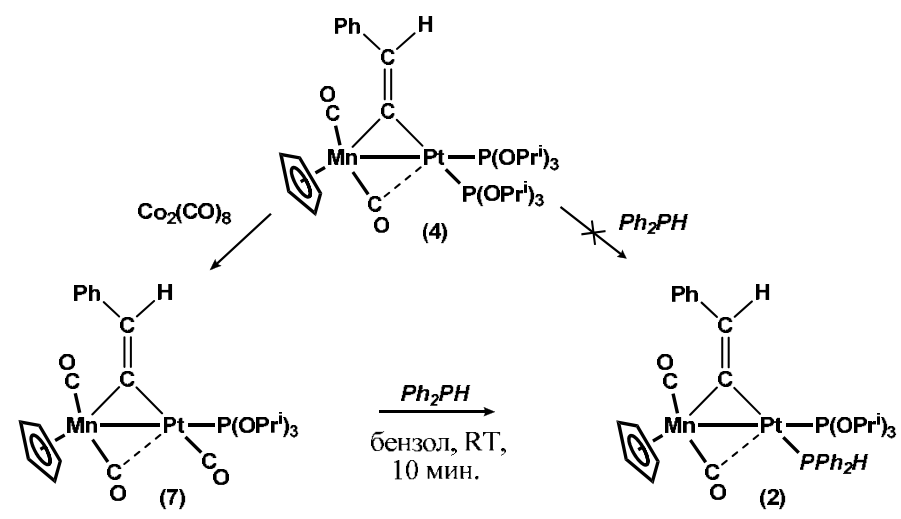

Рис. 2

Fig. 2
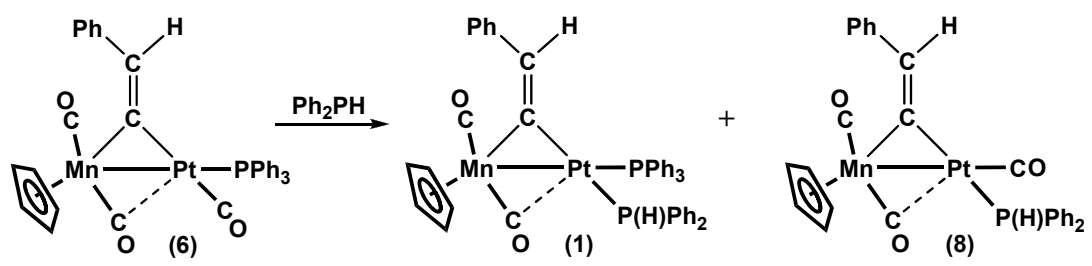

Рис. 3

Fig. 3

Однако в случае комплекса 6 реакция с дифенилфосфином протекает неселективно и приводит к замещению как карбонильного, так и трифенилфосфиного лигандов при атоме платины с образованием смеси продуктов, целевого комплекса 1 и необычного $\mathrm{Cp}(\mathrm{CO})_{2} \operatorname{MnPt}(\mu-$ $\mathrm{C}=\mathrm{CHPh})(\mathrm{CO})\left(\mathrm{Ph}_{2} \mathrm{PH}\right)(\mathbf{8})($ рис. 3).

Близкое значение $\mathrm{R}_{f}$ комплексов 1 и 8 затрудняет разделение смеси продуктов реакции методом колоночной хроматографии, поэтому эти соединения в индивидуальном виде были выделены с низкими выходами. Полученные соединения 1, 2 и 8 представляют собой желтые мелкокристаллические вещества, хорошо растворимые в полярных органических растворителях. Комплексы были изучены методами ЯМР- и ИК- спектроскопии.

Установлено, что наличие дифенилфосфиновых лигандов в координационном окружении атома платины полученных комплексов не оказывает значительного влияния на строение комплексов. В комплексах 1, 2 и 8 сохраняется мостиковая $\mu-\eta^{1}: \eta^{1}$-координация винилиденового лиганда и $\eta^{5}$-конфигурация циклопентадиенила с атомом марганца. Данные спектров ЯМР ${ }^{1} \mathrm{H}$ и ${ }^{13} \mathrm{C}$, в которых сигналы винилиденового и циклопентадиенильного лигандов находятся в таких же областях $\delta$, как и у исходных соединений 3, 4, 6 и 7 (табл. 1).

Изучение спектров ЯМР ${ }^{31}$ Р полученных соединений дает информацию о расположении фосфорсодержащих лигандов относительно друг друга в координационной сфере атома платины. Известно, что в биядерных винилиденовых MnPt комплексах атомы фосфора дифосфиновых лигандов неэквивалентны и находятся в разных положениях относительно $\mu$-винилиденового 
Таблица 1. Данные спектров ЯМР ${ }^{1} \mathrm{H},{ }^{31} \mathrm{P}$ и ${ }^{13} \mathrm{C}\left(\delta, \mathrm{ppm},[J\right.$, Гц] $)$ комплексов $\mathrm{Cp}(\mathrm{CO})_{2} \mathrm{Mn}[\mu-\mathrm{C}=\mathrm{CHPh}] \mathrm{Pt}(\mathrm{L})\left(\mathrm{L}{ }^{\prime}\right)$ $\left(\mathrm{L}=\mathrm{PPh}_{2} \mathrm{H}, \mathrm{L}^{\prime}=\mathrm{PPh}_{3}(\mathbf{1}) ; \mathrm{L}=\mathrm{PPh}_{2} \mathrm{H}, \mathrm{L}^{\prime}=\mathrm{P}\left(\mathrm{OPr}^{\mathrm{i}}\right)_{3}(\mathbf{2}) ; \mathrm{L}=\mathrm{PPh}_{2} \mathrm{H}, \mathrm{L}^{\prime}=\mathrm{CO}(\mathbf{8}) ; \mathrm{L}=\mathrm{L}^{\prime}=\mathrm{PPh}_{3}(\mathbf{3}) ; \mathrm{L}=\mathrm{L}^{\prime}=\mathrm{P}\left(\mathrm{OPr}^{\mathrm{i}}\right)_{3}\right.$ (4); $\left.\mathrm{L}=\mathrm{PPh}_{3}, \mathrm{~L}^{\prime}=\mathrm{CO}(\mathbf{6}) ; \mathrm{L}=\mathrm{P}\left(\mathrm{OPr}^{\mathrm{i}}\right), \mathrm{L}^{\prime}=\mathrm{CO}(7)\right)$

Table 1. NMR ${ }^{1} \mathrm{H},{ }^{31} \mathrm{P}$ и ${ }^{13} \mathrm{C}(\delta, \mathrm{ppm},[\mathrm{J}, \mathrm{Hz}])$ data of complexes $\mathrm{Cp}(\mathrm{CO})_{2} \mathrm{Mn}[\mu-\mathrm{C}=\mathrm{CHPh}] \mathrm{Pt}(\mathrm{L})\left(\mathrm{L}^{\prime}\right)\left(\mathrm{L}=\mathrm{PPh}_{2} \mathrm{H}\right.$, $\mathrm{L}^{\prime}=\mathrm{PPh}_{3}(\mathbf{1}) ; \mathrm{L}=\mathrm{PPh}_{2} \mathrm{H}, \mathrm{L}^{\prime}=\mathrm{P}\left(\mathrm{OPr}^{\mathrm{i}}\right)_{3}(\mathbf{2}) ; \mathrm{L}=\mathrm{PPh}_{2} \mathrm{H}, \mathrm{L}^{\prime}=\mathrm{CO}(\mathbf{8}) ; \mathrm{L}=\mathrm{L}^{\prime}=\mathrm{PPh}_{3}(\mathbf{3}) ; \mathrm{L}=\mathrm{L}^{\prime}=\mathrm{P}\left(\mathrm{OPr}^{\mathrm{i}}\right)_{3}(\mathbf{4})$; $\left.\mathrm{L}=\mathrm{PPh}_{3}, \mathrm{~L}^{\prime}=\mathrm{CO}(\mathbf{6}) ; \mathrm{L}=\mathrm{P}\left(\mathrm{OPr}^{\mathrm{i}}\right), \mathrm{L}^{\prime}=\mathrm{CO}(7)\right)$

\begin{tabular}{|c|c|c|c|c|c|c|c|}
\hline \multirow{3}{*}{ Комплекс } & \multicolumn{7}{|c|}{ ЯМР } \\
\hline & \multicolumn{3}{|c|}{${ }^{13} \mathrm{C}$} & \multicolumn{2}{|l|}{${ }^{1} \mathrm{H}$} & \multicolumn{2}{|c|}{${ }^{31} \mathrm{P}$} \\
\hline & $\mathrm{C}^{1}$ & $\mathrm{C}^{2}$ & $\mathrm{C}_{5} \mathrm{H}_{5}$ & $=\mathrm{C}^{2} \underline{\mathrm{H}} \mathrm{Ph}$ & $\mathrm{C}_{5} \mathrm{H}_{5}$ & $\mathrm{P}^{1}$ & $\mathrm{P}^{2}$ \\
\hline 1 & - & - & - & $\begin{array}{c}8,08 \text { д } \\
{ }^{4} J_{\mathrm{HP}}=11,0\end{array}$ & $4,31 \mathrm{c}$ & $\begin{array}{c}41,04 \text { д } \\
J_{\mathrm{PtP}}=4243 \\
{ }^{2} J_{\mathrm{PP}}=17,1\end{array}$ & $\begin{array}{c}5,74 \text { д } \\
J_{\mathrm{PtP}}=2470 \\
{ }^{2} J_{\mathrm{PP}}=17,1\end{array}$ \\
\hline 2 & $\begin{array}{c}261,2 \text { дд } \\
J_{\mathrm{PtCl}}=823,3 \\
2 J_{\mathrm{ClP}}=62,0\end{array}$ & $\begin{array}{c}140,2 \text { д } \\
{ }^{2} J_{\mathrm{PtC}}=129,1\end{array}$ & $84,6 \mathrm{c}$ & $\begin{array}{c}8,75 \text { дд } \\
{ }^{3} J_{\mathrm{PtH}}=30,1 \\
{ }^{4} J_{\mathrm{HP}}=14,9\end{array}$ & $4,43 \mathrm{c}$ & $\begin{array}{c}132,4 \text { д } \\
{ }^{2} J_{\mathrm{PP}}=11,4 \\
J_{\mathrm{PtP}}=6714\end{array}$ & $\begin{array}{c}4,25 \text { д } \\
{ }^{2} J_{\mathrm{PP}}=11,4 \\
J_{\mathrm{PtP}}=2252\end{array}$ \\
\hline 3 & $\begin{array}{c}264,30 \text { дд } \\
J_{\mathrm{PtCl}}=857,3 \\
{ }^{2} J_{\mathrm{ClP}}=61,4 ; 2,5\end{array}$ & $\begin{array}{c}141,25 \text { д } \\
{ }^{2} J_{\mathrm{PtC} 2}=114,5 \\
{ }^{3} J_{\mathrm{PC} 2}=6.7 \\
\end{array}$ & $84,19 \mathrm{c}$ & $\begin{array}{c}7.92 \text { д } \\
{ }^{3} J_{\mathrm{PtH}}=25,6 \\
{ }^{4} J_{\mathrm{PH}}=12,5\end{array}$ & $4,12 \mathrm{c}$ & $\begin{array}{c}43,5 \text { д } \\
2 J_{\mathrm{PP}}=17,1 \\
J_{\mathrm{PtP}}=4414\end{array}$ & $\begin{array}{c}27,4 \text { д } \\
{ }^{2} J_{\mathrm{PP}}=17,1 \\
J_{\mathrm{PtP}}=2607\end{array}$ \\
\hline 4 & $\begin{array}{c}259,48 \text { д } \\
J_{\mathrm{PtCl}}=817,5 \\
{ }^{2} J_{\mathrm{ClP}}=91,5\end{array}$ & $\begin{array}{c}141,36 \text { д } \\
{ }^{2} J_{\mathrm{PtC} 2}=117,8 \\
{ }^{3} J_{\mathrm{PC} 2}=6,5\end{array}$ & $84,40 \mathrm{c}$ & $\begin{array}{c}8,64 \text { д } \\
{ }^{3} J_{\mathrm{PtH}}=23,7 \\
{ }^{4} J_{\mathrm{PH}}=14,9 ; 20,2\end{array}$ & $4,68 \mathrm{c}$ & $\begin{array}{c}142,1 \text { д } \\
J_{\mathrm{PtP}}=6640 \\
{ }^{2} J_{\mathrm{PP}}=11\end{array}$ & $\begin{array}{c}145,3 \text { д } \\
J_{\mathrm{PtP}}=4020 \\
{ }^{2} J_{\mathrm{PP}}=11\end{array}$ \\
\hline 5 & $\begin{array}{c}259,97 \text { д } \\
J_{\mathrm{PtCl}}=858,6 \\
{ }^{2} J_{\mathrm{ClP}}=61,0\end{array}$ & $\begin{array}{c}140,27 \text { д } \\
{ }^{2} J_{\mathrm{PtC} 2}=122,0 \\
{ }^{3} J_{\mathrm{PC} 2}=6,0 \\
\end{array}$ & $83,82 \mathrm{c}$ & $\begin{array}{c}8,72 \text { дд } \\
{ }^{3} J_{\mathrm{PtH}}=16,8 \\
{ }^{4} J_{\mathrm{PH}}=15,2 ; 13,3\end{array}$ & $4,20 \mathrm{c}$ & $\begin{array}{c}133,9 \text { д } \\
J_{\mathrm{PtP}}=7048 \\
{ }^{2} J_{\mathrm{PP}}=11 \\
\end{array}$ & $\begin{array}{c}29,4 \\
J_{\mathrm{PtP}}=2356 \\
{ }^{2} J_{\mathrm{PP}}=11 \\
\end{array}$ \\
\hline 6 & $\begin{array}{c}260,48 \text { д } \\
J_{\mathrm{PtCl}}=916,9 \\
{ }^{2} J_{\mathrm{ClP}}=2,5\end{array}$ & $\begin{array}{c}146,34 \text { д } \\
{ }^{2} J_{\mathrm{PtC} 2}=123,4 \\
{ }^{3} J_{\mathrm{PC} 2}=4,5\end{array}$ & $85,05 \mathrm{c}$ & $\begin{array}{c}7,74 \text { д } \\
{ }^{3} J_{\mathrm{PtH}}=40,5 \\
{ }^{4} J_{\mathrm{PH}}=11,9\end{array}$ & $4,72 \mathrm{c}$ & $\begin{array}{c}36,5 \mathrm{c} \\
J_{\mathrm{PtP}}=3966\end{array}$ & \\
\hline 8 & - & - & - & $8,20 \mathrm{c}$ & $4,50 \mathrm{c}$ & & $\begin{array}{c}6,83 \text { д } \\
J_{\mathrm{PtP}}=2540 \\
\end{array}$ \\
\hline
\end{tabular}

лиганда [28]. Эта неэквивалентность отражается в их спектрах ЯМР ${ }^{31} \mathrm{P}$. В изученных ранее комплексах 3 и 4 сигнал в слабом поле, имеющий большую величину константы $J_{\mathrm{PtP}}$, был отнесен к атому $\mathrm{P}^{1}$, находящемуся в изис-положении к винилиденовому лиганду, а сигнал в сильном поле со значительно меньшей константой $J_{\mathrm{PtP}}$ к атому $\mathrm{P}^{2}$ в транс-положении к винилидену. В случае смешанолигандного комплекса $\mathrm{Cp}(\mathrm{CO})_{2} \mathrm{Mn}(\mu-\mathrm{C}=\mathrm{CHPh}) \mathrm{Pt}\left[\mathrm{P}\left(\mathrm{OPr}_{3}\right)_{3}\right]\left(\mathrm{PPh}_{3}\right)(5)$ наличие сигналов атома фосфора лиганда $\mathrm{PPh}_{3}$ при 29,4 м.д. с константой $J_{\mathrm{PtP}}=2356$ Гц и лиганда $\mathrm{P}\left(\mathrm{OPr}^{\mathrm{i}}\right)_{3}$ при 133,9 м.д. с константой $J_{\mathrm{PtP}}=7048$ Гц свидетельствует о транс-расположении лиганда $\mathrm{PPh}_{3}$ относительно винилидена [29].

В спектрах ЯМР ${ }^{31} \mathrm{P}$ новых комплексов 1 и 2 также наблюдаются неэквивалентные сигналы ядер фосфора. Характерные сигналы в слабом поле дифосфинового лиганда координированого с платиной [27] в комплексах 1 и 2 при 5,74 м.д. и 4,25 м.д., имеют константы $J_{\mathrm{PtP}} 2470$ и 2252 Гц, а константы $J_{\mathrm{PtP}}$ сигналов трифенилфосфинового (41,04 м.д.) и триизопропилфосфитного (132,4 м.д.) лигандов равны 4243 и 6714 Гц, соответственно (табл. 1). Такое соотношение констант $J_{\mathrm{PtP}}$ свидетельствует о том, что дифенилфосфиновые лиганды при атоме платины в обоих комплексах 1 и 2 расположены в транс-положении относительно винилидена, тогда как лиганды $\mathrm{PPh}_{3}$ и $\mathrm{P}\left(\mathrm{OPr}^{\mathrm{i}}\right)_{3}$ в ųuс-положении к нему. 
В спектре ЯМР ${ }^{31} \mathrm{P}$ изученного ранее трикарбонильного комплекса $\mathrm{Cp}(\mathrm{CO})_{2} \mathrm{Mn}(\mu-\mathrm{C}=\mathrm{CHPh})$ $\mathrm{Pt}\left(\mathrm{PPh}_{3}\right)(\mathrm{CO})(6)$ содержится синглет с константой $J_{\mathrm{PtP}}=3966$ Гц, что свидетельствует о изисположении лиганда $\mathrm{PPh}_{3}$ относительно винилидена [29]. В случае же нового трикарбонильного комплекса $\mathrm{Cp}(\mathrm{CO})_{2} \mathrm{Mn}(\mu-\mathrm{C}=\mathrm{CHPh}) \mathrm{Pt}(\mathrm{CO})\left(\mathrm{PPh}_{2} \mathrm{H}\right)(8)$ значение константы $J_{\mathrm{PtP}}$ при 6,83 м.д. составляет 2540 Гц и указывает на транс-ориентацию дифенилфосфинового лиганда (табл. 1). Таким образом, в 8 карбонильная группа при атоме платины находится в транс-положении к атому марганца, а $\mathrm{PPh}_{2} \mathrm{H}$ лиганд - в транс-положении к винилидену. Следует отметить, что такое расположение лигандов при атоме Рt является не вполне типичным [29, 30].

ИК спектры комплексов 1 и 2 в области валентных колебании СО-групп содержат две полосы, соответствующие колебаниям карбонильных групп, координированных с атомом марганца (табл. 2). Ранее [29, 31] было показано, что в биядерных MnPt комплексах эти группы различаются координацией с соседними атомами металлов, так высокочастотная полоса относится к терминальной $\mathrm{CO}$-группе $\left(\mathrm{CO}_{\mathrm{T}}\right)$, тогда как низкочастотная соответствует полумостиковой карбонильной группе $\left(\mathrm{CO}_{\text {пм }}\right)$, дополнительно связанной с соседним атомом платины за счет перекрывания незаполненных $\pi^{*}$ орбиталей СО-группы с заполненными d-орбиталями платины (рис. 4).

При этом с увеличением электронодонорной способности лигандов L при атоме Pt и уменьшением создаваемых ими стерических препятствий степень полумостикого взаимодействия усиливается, становясь практически мостиковой (резонансный тип II на рис. 4). Характеристи-

Таблица 2. Данные ИК спектров комплексов $\mathrm{Cp}(\mathrm{CO}){ }_{2} \operatorname{MnPt}(\mu-\mathrm{C}=\mathrm{CHPh})(\mathrm{L})\left(\mathrm{L}^{\prime}\right)$

Table 2. IR data of complexes $\mathrm{Cp}(\mathrm{CO})_{2} \operatorname{MnPt}(\mu-\mathrm{C}=\mathrm{CHPh})(\mathrm{L})\left(\mathrm{L}^{\prime}\right)$

\begin{tabular}{|c|c|c|}
\hline Комплекс & $v(\mathrm{CO}), \mathrm{CH}_{2} \mathrm{Cl}_{2}, \mathrm{~cm}^{-1}$ & $\Delta v(\mathrm{CO}), \mathrm{cm}^{-1}$ \\
\hline $\mathrm{Cp}(\mathrm{CO})_{2} \operatorname{MnPt}(\mu-\mathrm{C}=\mathrm{CHPh})\left(\mathrm{PPh}_{2} \mathrm{H}\right)\left(\mathrm{PPh}_{3}\right)(\mathbf{1})$ & 1920,1828 & 92 \\
\hline $\mathrm{Cp}(\mathrm{CO})_{2} \operatorname{MnPt}(\mu-\mathrm{C}=\mathrm{CHPh})\left(\mathrm{PPh}_{2} \mathrm{H}\right)\left[\mathrm{P}\left(\mathrm{OPr}^{\mathrm{i}}\right)_{3}\right](\mathbf{2})$ & 1923,1828 & 95 \\
\hline $\mathrm{Cp}(\mathrm{CO})_{2} \mathrm{MnPt}(\mu-\mathrm{C}=\mathrm{CHPh})\left(\mathrm{PPh}_{3}\right)_{2}(\mathbf{3})$ & 1924,1838 & 86 \\
\hline $\mathrm{Cp}(\mathrm{CO})_{2} \mathrm{MnPt}(\mu-\mathrm{C}=\mathrm{CHPh})\left[\mathrm{P}\left(\mathrm{OPr}^{2}\right)_{3}\right]_{2}(\mathbf{4})$ & 1929,1863 & 66 \\
\hline $\mathrm{Cp}(\mathrm{CO})_{2} \operatorname{MnPt}(\mu-\mathrm{C}=\mathrm{CHPh})\left[\mathrm{P}(\mathrm{OPr})_{3}\right]\left(\mathrm{PPh}_{3}\right)(\mathbf{5})$ & 1926,1842 & 84 \\
\hline $\mathrm{Cp}(\mathrm{CO})_{2} \operatorname{MnPt}(\mu-\mathrm{C}=\mathrm{CHPh})(\mathrm{CO})\left(\mathrm{PP}_{3}\right)(\mathbf{6})$ & $2032,1938,1871$ & 67 \\
\hline $\mathrm{Cp}(\mathrm{CO})_{2} \operatorname{MnPt}(\mu-\mathrm{C}=\mathrm{CHPh})(\mathrm{CO})\left[\mathrm{P}\left(\mathrm{OPr}^{\mathrm{i}}\right)_{3}\right](\mathbf{7})$ & $2045,1928,1867$ & 61 \\
\hline
\end{tabular}
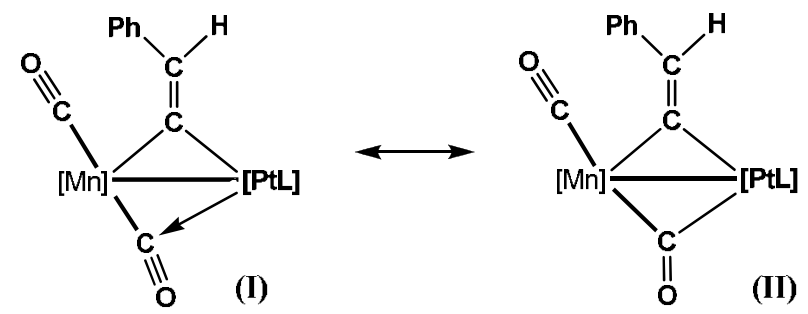

Рис. 4

Fig. 4 
кой степени взаимодействия $\mathrm{CO}_{\text {пм} \cdots} \cdots \mathrm{Pt}$ является величина $\Delta v(\mathrm{CO})=v\left(\mathrm{CO}_{\mathrm{\tau}}\right)-v\left(\mathrm{CO}_{\text {пм }}\right)$, соответствующая разности частот валентных колебаний терминальной и полумостиковой СО-групп [31].

Так как б-донорная способность дифенилфосфинового лиганда меньше трифенилфосфинового, но при этом немного больше триизопропилфосфитного, то в случае замены $\mathrm{Ph}_{3} \mathrm{P}-$ лиганда на $\mathrm{Ph}_{2} \mathrm{PH}$ следовало бы ожидать сдвига $v(\mathrm{CO})_{\text {п }}$ в высокочастотную область, а при замене $\left(\mathrm{OPr}^{\mathrm{i}}\right)_{3} \mathrm{P}$-лиганда на $\mathrm{Ph}_{2} \mathrm{PH}$ - незначительного сдвига в низкочастотную область. Однако в ИК спектрах комплексов 1 и 2 наблюдается противоположная картина - частоты $v(\mathrm{CO})_{\text {пм }}$ значительно сдвинуты в низкочастотную область, а значение $\Delta v(\mathrm{CO})$ увеличено относительно

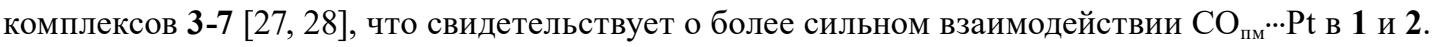
Таким образом, в случае комплексов 1 и 2 решающим фактором увеличения полумостикового взаимодействия является не электронодонорная способность дифенилфосфиновых лигандов при атоме платины, а их меньший конический угол $\left(\theta=128^{\circ}\right)$ по сравнению с $\mathrm{Ph}_{3} \mathrm{P}\left(\theta=145^{\circ}\right)$ и $\left(\mathrm{OPr}^{\mathrm{i}}\right)_{3} \mathrm{P}\left(\theta=130^{\circ}\right)$ [32], что приводит к большему взаимодействию СО-группы с соседним атомом платины.

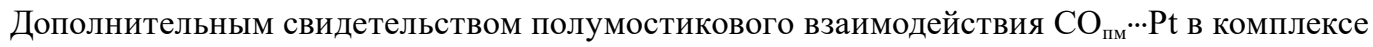
2 является наличие в спектре ЯМР ${ }^{13} \mathrm{C}$, снятом при $-35^{\circ} \mathrm{C}$, расщепления сигнала в слабом поле (236.99 м.д.) одной из карбонильных групп на ядрах фосфора и платины с константами $J_{\mathrm{PC}}$ и $J_{\mathrm{PtC}}, 12.7$ и 104 Гц, соответственно. Следует также отметить, что при комнатной температуре спектр ЯМР ${ }^{13} \mathrm{C}$ комплекса 2 содержит два широких сигнала СО при 236.43 и 229.48 м.д., а последовательное понижение температуры до $-35{ }^{\circ} \mathrm{C}$ приводит к появлению двух четких сигналов карбонильных групп, узкого синглета (229.78 м.д.) группы $\mathrm{CO}_{\text {т }}$ и характерного дублета

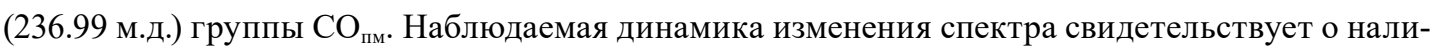

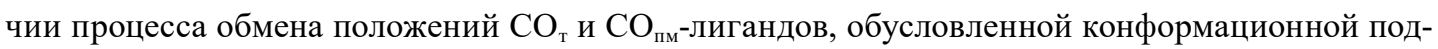
вижностью фрагмента $\left[\mathrm{Cp}(\mathrm{CO})_{2} \mathrm{Mn}\right]$ (рис. 5). Такие процессы наблюдались ранее [33, 34], в том числе для аналогичных биядерных RePt комплексов [22].

Таким образом, в настоящей работе разработаны методики синтеза новых биядерных комплексов $\mathrm{Cp}(\mathrm{CO})_{2} \operatorname{MnPt}(\mu-\mathrm{C}=\mathrm{CHPh})\left(\mathrm{PPh}_{2} \mathrm{H}\right)(\mathrm{L})\left(\mathrm{L}=\mathrm{PPh}_{3}(\mathbf{1}), \mathrm{P}\left(\mathrm{OPr}^{i}\right)_{3}(\mathbf{2}),(\mathrm{CO})(\mathbf{8})\right)$. На основании анализа их ИК и ЯМР ${ }^{1} \mathrm{H},{ }^{31} \mathrm{P},{ }^{13} \mathrm{C}$ спектров и сравнения с данными ранее синтезированных комплексов 3 - 7, предложено строение полученных соединений 1, 2 и 8. Установлено, что введение дифенилфосфинового лиганда в координационную сферу атома платины не приводит к значительным изменениям строения комплексов 1 и 2 , сохраняется мостиковая $\mu-\eta^{1}: \eta^{1}$-координация винилиденового лиганда и $\eta^{5}$-конфигурация циклопентадиенила с атомом марганца. Однако вследствие меньших стерических затруднений, создаваемых лигандом $\mathrm{Ph}_{2} \mathrm{PH}$ в комплексах $\mathbf{1}$, 2, наблюдается значительное усиление взаимодействия между полумостиковой карбонильной группой $\mathrm{CO}_{\text {пм }}$ при атоме $\mathrm{Mn}$ и соседним атомом Pt по сравнению с раннее изученными соединениями 3-5. 


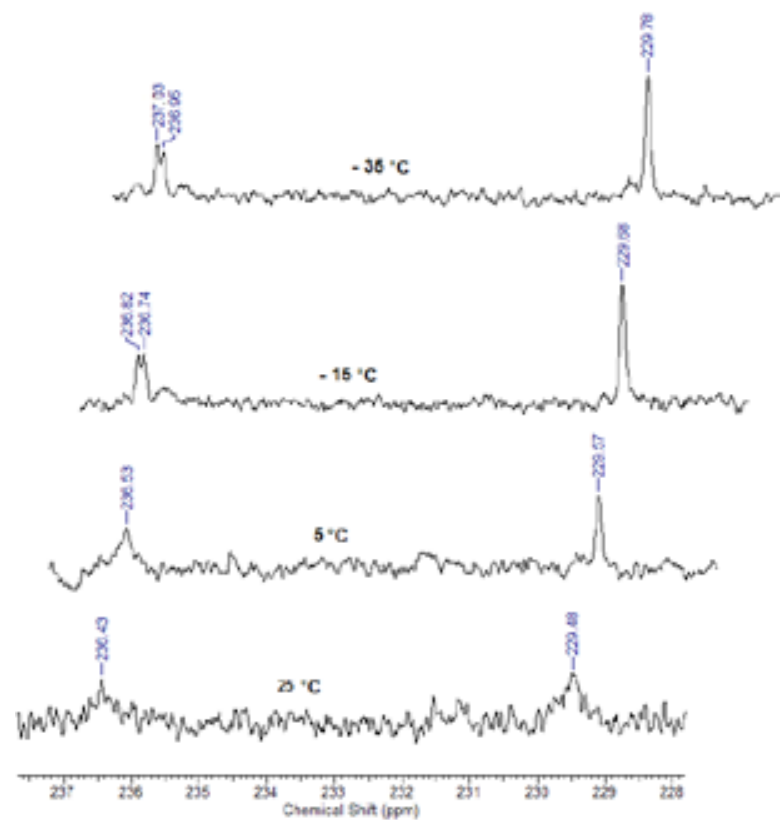

Рис. 5. Спектр ЯМР ${ }^{13} \mathrm{C}$ комплекса $\mathrm{Cp}(\mathrm{CO})_{2} \mathrm{Mn}(\mu-\mathrm{C}=\mathrm{CHPh}) \mathrm{Pt}\left(\mathrm{PPh}_{2} \mathrm{H}\right)\left[\mathrm{P}\left(\mathrm{OPr}^{\mathrm{i}}\right)_{3}\right]$ (2) при различных температурах

Fig. 5. VT $N M R{ }^{13} \mathrm{C}$ spectrum of complex $\mathrm{Cp}(\mathrm{CO})_{2} \mathrm{Mn}(\mu-\mathrm{C}=\mathrm{CHPh}) \mathrm{Pt}\left(\mathrm{PPh}_{2} \mathrm{H}\right)\left[\mathrm{P}\left(\mathrm{OPr}^{\mathrm{i}}\right)_{3}\right]$ (2)

\section{Список литературы}

1. Sculfort S., Braunstein P. Intramolecular d10-d10 interactions in heterometallic clusters of the transition metals. Chem. Soc. Rev 2011. Vol. 40(5), P. 2741-2760.

2. Adams R.D. Metal segregation in bimetallic clusters and its possible role in synergism and bifunctional catalysis. J. Organomet. Chem. 2000. Vol. 600(1-2), P. 1-6.

3. Xiao J., Puddephatt R.J. Pt-Re clusters and bimetallic catalysts. Coord. Chem.. Rev 1995. Vol. 143, P. 457-500.

4. Ungvary F. Application of transition metals in hydroformylation. Annual survey covering the year 2000. Coord. Chem. Rev. 2001. Vol. 218, P. 1-41.

5. Thomas J.M., Johnson B.F.G., Raja R., Sankar G., Midgley P.A. High-Performance Nanocatalysts for Single-Step Hydrogenations. Acc. Chem. Res. 2003. Vol. 36(1), P. 20-30.

6. Werner H. Vinylidenerhodium complexes as promising tools for $\mathrm{C}-\mathrm{C}$ coupling reactions. Coord. Chem. Rev. 2004. Vol. 248(15-16), P. 1693-1702.

7. Ritleng V., Chetcuti M.J.Hydrocarbyl Ligand Transformations on Heterobimetallic Complexes. Chem. Rev. 2007. Vol. 107(3), P. 797-858.

8. Wheatley N., Kalck P. Structure and Reactivity of Early-Late Heterobimetallic Complexes. Chem. Rev. 1999. Vol. 99(12), P. 3379-3420.

9. Adams R.D., Captain B. Hydrogen Activation by Unsaturated Mixed-Metal Cluster Complexes: New Directions. Angew. Chemie Int. Ed. 2008. Vol. 47(2), P. 252-257.

10. Able E.W., Stone F.G.A., Wilkinson G. Comprehensive Organometallic Chemistry: Heteronuclear metal-metal bonds. Vol. 10 / ed. Adams R.D. Pergamon, 1995. 423 p. 
11. Adams R.D., Boswell E.M., Hall M.B., Yang X. Activation of Hydrogen and Related Small Molecules by the $\mathrm{PtOs}_{3}(\mathrm{CO})_{7}\left(\mathrm{P}-t-\mathrm{Bu}_{3}\right)\left(\mu-\mathrm{P}-t-\mathrm{Bu}_{2}\right)\left(\mu_{4}-\mathrm{CHCMeCH}\right)$. Organometallics 2008. Vol. 27, P. 4938-4947.

12. Karunananda M.K., Mankad N.P. E-Selective Semi-Hydrogenation of Alkynes by Heterobimetallic Catalysis. J. Am. Chem. Soc. 2015. Vol. 137(46), P. 14598-14601.

13. Bruce M.I. Organometallic chemistry of vinylidene and related unsaturated carbenes. Chem. Rev. 1991. Vol. 91(2), P. 197-257.

14. Zhang L., Zhu B., Xiao N., Xu Q., Tsumori N., Sun J., Yin Y., Chen J. Remarkable Reactions of Cationic Carbyne Complexes of Manganese and Rhenium with the Carbonylrhodium Anion $\left[\mathrm{Rh}(\mathrm{CO})_{4}\right]^{-}$. A Novel Route to Heteropolymetallic Bridging Carbyne Complexes with $\mu$ - and $\mu_{3}$-Carbyne Ligands. Organometallics 2003. Vol. 22(22), P. 4369-4371.

15. Werner H., Alonso F.J.G., Otto H., Peters K., Von Schnering H.G. Vinylidene Transition-metal complexes, VI. The rhodium compounds $\mathrm{C}_{5} \mathrm{H}_{5} \mathrm{Rh}(=\mathrm{C}=\mathrm{CHR}) \mathrm{PiPr}_{3}$ as building blocks for the synthesis of heterometallic Di- and trinuclear vinylidene-bridged complexes. Chem. Ber. 1988. Vol. 121(9), P. 1565-1573.

16. Berenguer J.R., Lalinde E., Teresa Moreno M. An overview of the chemistry of homo and heteropolynuclear platinum complexes containing bridging acetylide $(\mu-\mathrm{C} \equiv \mathrm{CR})$ ligands. Coord. Chem. Rev. 2010. Vol. 254(7-8), P. 832-875.

17. Stone F.G.A. Zero-valent platinum complexes and their role in the synthesis of di-, tri-, and tetra-nuclear metal compounds. Inorg. Chim. Acta 1981. Vol. 50, P. 33-42.

18. Bruce M.I. Transition Metal Complexes Containing Allenylidene, Cumulenylidene, and Related Ligands. Chem. Rev. 1998. Vol. 98(8), P. 2797-2858.

19. Antonova A.B. Use of the $\mathrm{Mn}=\mathrm{C}=\mathrm{C}$ system in organometallic and organic synthesis. Coord. Chem. Rev. 2007. Vol. 251(11-12), P. 1521-1560.

20. Antonova A.B., Chudin O.S.,, Vasiliev A.D., Pavlenko N.I., Sokolenko W.A., Rubaylo A.I., Semeikin O.V. Chemistry of vinylidene complexes. XVIII. Synthesis and molecular structure of the novel trinuclear $\mu_{3}$-vinylidene complex $\mathrm{CpReFePt}\left(\mu_{3}-\mathrm{C}=\mathrm{CHPh}\right)(\mathrm{CO})_{6}\left(\mathrm{PPh}_{3}\right)$. J. Organomet. Chem. 2009. Vol. 694(1), P. 127-130.

21. Antonova A.B., Chudin O.S., Pavlenko N.I., Sokolenko W.A., Rubaylo A. I., Vasiliev A.D., Verpekin V.V., Semeikin O.V. Chemistry of vinylidene complexes 19. New heterometallic $\mu$-vinylidene complexes with $\mathrm{Re}-\mathrm{M}(\mathrm{M}=\mathrm{Pd}, \mathrm{Pt})$ bonds. Molecular structure of $\left(\eta-{ }^{5} \mathrm{C}_{5} \mathrm{H}_{5}\right)(\mathrm{CO}) \operatorname{RePt}(\mu-\mathrm{C}=\mathrm{CHPh})$ ( $\mu-\mathrm{CO})\left(\mathrm{Ph}_{2} \mathrm{PCH}_{2} \mathrm{PPh}_{2}\right)$. Russian Chemical Bulletin, Int. Ed. 2009, Vol. 58(5), P. 955-963.

22. Antonova A.B., Verpekin V.V., Chudin O.S., Vasiliev A.D., Pavlenko N.I., Sokolenko W.A., Rubaylo A.I., Semeikin O.V. Chemistry of vinylidene complexes. XXI. Synthesis, spectroscopic and structural study of the RePt and MnPt $\mu$-vinylidene complexes. Inorganica Chim. Acta 2013. Vol. 394, P. 328-336.

23. Verpekin V.V., Kondrasenko A.A., Chudin O.S., Vasiliev A.D., Burmakina G.V., Pavlenko N.I., Rubaylo A.I. Chemistry of vinylidene complexes. XXIII. Binuclear rhenium-palladium vinylidene bridged complexes, their reactions with diiron nonacarbonyl. J. Organomet. Chem. 2014. Vol. 770, P. $42-50$.

24. Верпекин В.В., Чудин О.С., Пирязев Д.А., Рубайло А.И., Громилов С.А., Семейкин О.В. Новый трехъядерный $\mu_{3}$-винилиденовый кластер $\mathrm{CpReFePt}\left(\mu_{3}-\mathrm{C}=\mathrm{CHPh}\right)(\mathrm{CO})_{5}\left[\mathrm{P}\left(\mathrm{OPr}^{\mathrm{i}}\right)_{3}\right]_{2}$. Жур- 
нал структурной химии 2015. T. 56(4), C. 823-825. [Verpekin V.V., Chudin O.S., Piryzev D.A., Rubaylo A.I., Gromilov S.A., Semeikin O.V. New trinuclear $\mu_{3}$-vinylidene cluster $\operatorname{CpReFePt}\left(\mu_{3^{-}}\right.$ $\mathrm{C}=\mathrm{CHPh})(\mathrm{CO})_{5}\left[\mathrm{P}\left(\mathrm{OPr}^{\mathrm{i}}\right)_{3}\right]_{2}$. Russian journal of structural chemistry 2015. Vol. 56(4), P. 823-825. (In Russ.)]

25. Powell J., Couture Ch., Gregg M.R., Sawyer J.F. Binuclear Phosphido-Bridged CrPt, MoPt, and WPt Complexes. Synthesis of $(\mathrm{CO})_{4} \mathrm{M}\left(\mu-\mathrm{PPh}_{2}\right)_{2} \mathrm{Pt}\left(\mathrm{PR}_{3}\right)$ Complexes and Effect of the $\mathrm{M} \rightarrow \mathrm{Pt}$ Donor Bond on the Reactivity of the Carbonyl Ligands. Inorg. Chem. 1989. Vol. 28, P. 3437-3444.

26. Alonso E., Forniès J., Fortuño C., Martín A., Orpen A.G. Reactivity of a Secondary Phosphine Platinum(II) Complex with [Pt(norbornene) $)_{3}$ and $\mathrm{PPh}_{3}$. Synthesis of New Single Phosphido-Bridged Derivatives of Platinum(I) and Phosphido-Bridged Platinum(II) Hydrides. Organometallics 2001. Vol. 20, P. 850-859.

27. Falvello L.R., Forniès J., Martín A., Gómez J., Lalinde E., Moreno M.T., Sacristán J. Synthesis of Heterobridged $(\mu-\mathrm{C} \equiv \mathrm{CR})(\mu-\mathrm{X})\left(\mathrm{X}=\mathrm{PPh}_{2}, \mathrm{PPh}_{2} \mathrm{O}\right)$ Platinum-Rhodium or Platinum-Iridium Dimers. Inorg. Chem.1999. Vol. 38, P. 3116-3125.

28. Antonova A.B., Kovalenko S.V., Korniyets E.D., Petrovsky P.V., Gulbis G.R., Johansson A.A. Chemistry of Vinylidene Complexes. III. Binuclear Manganese-Platinum Complexes with Bridging Phenylvinylidene Ligand. Inorganica Chim. Acta 1985. Vol. 96, P. 1-7.

29. Antonova A.B., Kovalenko S.V., Korniyets E.D., Petrovsky P.V., Johansson A.A., Deykhina N.A. Chemistry of vinylidene complexes. V. The ligand substitution reactions at the platinum atom in complexes $\mathrm{Cp}(\mathrm{CO})_{2} \mathrm{MnPt}(\mu-\mathrm{C}=\mathrm{CHPh}) \mathrm{L}_{2}$. Inorganica Chim. Acta 1985. Vol. 105(2), P. 153-163.

30. Verpekin V.V. Vasiliev A.D., Kondrasenko A.A., Burmakina G.V., Chudin O.S., Pavlenko N.I., Zimonin D.V., Rubaylo A.I. Chemistry of vinylidene complexes. XXIV. A new $\mu$-vinylidene complex containing RePt core, and platinum-bound carbonyl ligand. Spectroscopic, structural and electrochemical study. J. Mol. Structure 2018. Vol. 1163, P. 308-315.

31. Johansson A.A., Antonova A.B., Pavlenko N.I., Rubailo A.I. Infrared study of transformation of a terminal carbonyl ligand into a bridging one in the $\mathrm{MnPt}$ and $\mathrm{MnPd} \mu$-vinylidene complexes. $J$. Mol. Structure 1997. Vol. 408/409, P. 329-332.

32. Tolman C.A. Steric Effects of Phosphorus Ligands in Organometallic Chemistry and Homogeneous Catalysis. Chem. Rev. 1977. Vol. 77(3), P. 313-348.

33. Dawkins G.M., Green M., Jeffery J.C., Sambale C., Stone F.G.A. Formation of a bridged vinylidenedi-iron complex by nucleophilic attack on a carbonyl ligand of $\left[\mathrm{Fe}_{2}(\mathrm{CO})_{4}\left(\eta-\mathrm{C}_{5} \mathrm{H}_{5}\right)_{2}\right]$, and the synthesis and $X$-ray crystal structure of $\left[(\mathrm{OC})_{3} \mathrm{Fe}\left(\left(\mu-\mathrm{Ph}_{2} \mathrm{PCHPPh}_{2}\right) \mathrm{FeH}(\mathrm{CO})_{3}\right]\right.$. J. Chem. Soc. Dalton Trans. 1983. P. 499-506.

34. Colborn R.E., Davies D.L., Dyke A.F., Knox S.A.R., Mead K.A., Orpen A.G. Guerchais J.E., Roué J. Organic chemistry of dinuclear metal centres. Part 12. Synthesis, $X$-ray crystal structure, and reactivity of the di- $\mu$-alkylidene complex $\left[\mathrm{Ru}_{2}(\mathrm{CO})_{2}(\mu-\mathrm{CHMe})\left(\mu-\mathrm{CMe}_{2}\right)\left(\eta-\mathrm{C}_{5} \mathrm{H}_{5}\right)_{2}\right]$ : alkylidene linking. $J$. Chem. Soc. Dalton Trans. 1989. P. 1799-1805. 